\title{
Determination of the $\pi^{\circ} \gamma$ Cross Section
}

\author{
G. Govi, M. Lombardo and F. Marchetto
}

I.N.F.N. and University of Turin, 10125 Turin, Italy

\section{Introduction}

In this memo we address the following questions: how the $\pi^{\circ} \gamma$ cross section, which is measured in E760, compares to the expected leakage from the $\pi^{\circ} \pi^{0}$ final states? is it possible to affirm that a substantial fraction of the $\pi^{\circ} \gamma$ events are due to continuum production?

Naively, the expected continuum $\pi^{\circ} \gamma$ cross section can be estimated using the Vector Meson Dominance Model (VDM). In that framework, a photon is coupled to a neutral vector meson $V$ by $G_{\gamma V}$. Then $\sigma_{\pi \sigma_{\gamma}}=\sigma_{\pi^{\circ} \rho} \times G_{\gamma \rho}^{2}$ with $G_{\gamma \rho}^{2}=1 . / 160 \div 1 . / 380[1]$.

$184 \div 438 \mathrm{nb}$

At $E_{C M}=2.611 \mathrm{GeV}, \sigma_{\pi^{\circ} \rho}=(70 \pm 30) \mu \mathrm{b}[2]$ which gives $\sigma_{\pi^{\circ} \gamma}=$

To predict the cross section within a limited acceptance we assume that the angular production of $\pi^{\circ} \gamma$ is similar to the $\pi^{\circ} \pi^{\circ}$ and $\pi^{\circ} \rho$ ones. To estimate the fraction of events with $\left|\cos \theta^{*}\right|<0.5$, being $\theta^{*}$ the center of mass production angle, we do use the total $\pi^{+} \pi^{-}$cross section $\sigma_{\pi^{+} \pi^{-}}=$ $(6.6 \pm 3.5) \mu \mathrm{b}$ at $2.975 \mathrm{GeV}[3] . \sigma_{\pi^{0} \pi^{\circ}}=0.5 \times \sigma_{\pi^{+} \pi^{-}}$, while for $\left|\cos \theta^{*}\right|<0.5$ E760 measures $\sigma_{\pi^{\circ} \pi^{\circ}} \simeq 180 \mathrm{nb}$ at the above center of mass energy and $\left|\cos \theta^{*}\right|<0.5$. We obtain that the fraction of $\pi^{\circ} \pi^{\circ}$ events with $\left|\cos \theta^{*}\right|<0.5$ is $5.4 \times 10^{-2}$. Finally, multiplying the expected total cross section and the above fraction, we obtain $\sigma_{\pi^{*} \gamma_{\gamma}}=(10 . \div 23.8)$ nb with $\left|\cos \theta^{*}\right|<0.5$. This prediction gives a value which is far from being negligible and certainly measurable in our experiment.

To extract $\sigma_{\pi^{\circ} \gamma}$ as a function of $\cos \theta^{*}$ from E760 data, we use the following relationships valid for each interval of $\cos \theta^{*}$ :

$$
\frac{d \sigma_{\pi^{0} \pi^{0}}^{M}}{d \cos \theta^{*}}=P_{\pi^{0} \pi^{0}}^{\pi^{0}} \frac{d \sigma_{\pi^{0} \pi^{0}}}{d \cos \theta^{*}}
$$




$$
\begin{gathered}
\frac{d \sigma_{\pi^{0} \gamma}^{M}}{d \cos \theta^{*}}=P_{\pi^{0} \pi^{0}}^{\pi^{0}} \frac{d \sigma_{\pi^{0} \pi^{0}}}{d \cos \theta^{*}}+P_{\pi^{0} \gamma}^{\pi^{0} \gamma} \frac{d \sigma_{\pi^{0} \gamma}}{d \cos \theta^{*}} \\
\frac{d \sigma_{\gamma \gamma}^{M}}{d \cos \theta^{*}}=P_{\pi^{0} \pi^{0}}^{\gamma \gamma} \frac{d \sigma_{\pi^{0} \pi^{0}}}{d \cos \theta^{*}}+P_{\pi^{0} \gamma}^{\gamma \gamma} \frac{d \sigma_{\pi^{0} \gamma}}{d \cos \theta^{*}}+P_{\gamma \gamma}^{\gamma \gamma} \frac{d \sigma_{\gamma \gamma}}{d \cos \theta^{*}}
\end{gathered}
$$

where $d \sigma^{M} / d \cos \theta^{m}$ represent the measured cross sections, $d \sigma / d \cos \theta^{*}$ are the corrected cross sections, and $P_{X}^{Y}$ gives the probability that a final state $X$ is misinterpreted and reconstructed as $Y . P_{X}^{Y}$ comes from the contribution of several terms:

a) trigger efficiency $\epsilon_{t}$ : probability that given an event $X$, it goes through the trigger. It has been measured $\epsilon_{t}=(0.87 \pm 0.03)$ [4] for $\pi^{\circ} \pi^{\circ}$, and from that it has been estimated: $\epsilon_{t}=(0.89 \pm 0.03)$ for $\pi^{\circ} \gamma$ and $\epsilon_{t}=(0.91 \pm 0.03)$ for $\gamma \gamma$;

b) first pass data reduction efficiency $\epsilon_{f p}$. Typically, we used the ACP bits to make a first selection of the events to speed up the analysis, and to measure $\epsilon_{p p}$ we processed the data without the ACP cut on a limited part of the full sample;

c) analysis efficiency $\epsilon_{a n}$, which includes final selection and fit efficiencies;

d) acceptance and cluster reconstruction efficiency, $\epsilon_{\text {acc }}$, which takes into account the leakage probability from $X$ to $Y$, due to $\gamma$ 's escaping the detection (out of the detector or below energy threshold) or coalescent pairs which are not separated by the clustering algorithm. Both cause a wrong number of reconstructed $\gamma$ 's in the final state. We made extensive use of a Monte Carlo to evaluate $\epsilon_{a n} \times \epsilon_{a c e}$ as a function of $E_{C M}$ and $\cos \theta^{*}$. The Monte Cario is described in some detail in the next section.

For the preseat analysis we assumed that the MLU4 DST selection didn't introduce any inefficiency for the considered channels. Furthermore, we limited the acceptance to $\left|\cos \theta^{*}\right|<0.5$ for all the channels to avoid delicate effect of the acceptance on the sample consistency.

Finally, we mention that we used as energy thresholds $5 \mathrm{MeV}$ for the central block and $20 \mathrm{MeV}$ for the total energy of the cluster.

\section{Monte Carlo description}

The Monte Carlo [5] consists of an event generator and a shower simulator. It has been integrated in the offine package and Monte Carlo events go thru the same routines as E760 data.

The event generator is rather standard and uses the CERN package GENBOD. The shower simulator (not fully implemented for the Forward Calorimeter) is based on the parametrization of the lateral development of the shower, which is described by a double exponential [6]. The amount of 
energy $d E$ deposited on an elementary area $d x d y$ of the Central Calorimeter is given by (in arbitrary units):

$d E=\left(A_{1} \exp \frac{-\left|x-x_{0}\right|}{\lambda_{1}}+B_{1} \exp \frac{-\left|x-x_{0}\right|}{\lambda_{2}}\right) \times\left(A_{2} \exp \frac{-\left|y-y_{0}\right|}{\lambda_{3}}+B_{2} \exp \frac{-\left|y-y_{0}\right|}{\lambda_{4}}\right) \times d x d y$

where $x-x_{0}$ and $y-y_{0}$ are the distances of the elementary area from the shower center position in terms of counter fraction along the ring and wedge respectively, $\lambda_{1}=0.03208, \lambda_{2}=0.1860, \lambda_{3}=0.03969, \lambda_{4}=0.1715$ and $A_{1}=$ $A_{2}=10 ., B_{1}=1.706, B_{2}=1.4522$.

The energy deposited on a counter is the result of the integration of $d E$ over the counter area. The integration was then performed over a $3 \times 3$ grid around the shower position. Cracks were taken into account limiting the integration to the counter active areas. Effectively, this is an approximation because the steal and the lead glass have different absorption length, but a partial correction stays in the overall energy normalization. Finally the energy deposited $E$ on each block was randomly dispersed according to a Gaussian distribution with r.m.s. given by: $\sigma_{E}=0.035 \times \sqrt{E}+0.05 \times E$.

Monte Carlo and experimental data are in a good agreement. For a $\pi^{\circ} \pi^{\circ}$ final state, fig 1.a (b) shows the total reconstructed energy for Monte Carlo (data). Fig 2a (b) reports the difference between the reconstructed and the fitted $\gamma$ polar angle for Monte Carlo (data) and in fig. 3.a (b) for the azimuthal angle.

The cluster mass (as defined in [4]) and the reconstructed $\pi^{\circ}$ mass are equally well simulated, as shown in fig. 4 and 5 .

\section{Event Selection}

a) $\pi^{\circ} \pi^{\circ}$ selection

As input we used the $\pi^{\circ} \pi^{\circ}$ files produced by G. Zioulas during the 91 data taking. We measured the efficiency of the G.Z. first pass selection comparing the $\pi^{\circ} \pi^{\circ}$ sample obtained by using as input MLU4 DST and the sample from G.Z. files. We obtained $\epsilon_{p}=0.87$, which includes also the effect due to the different thresholds used for the clusterization.

The final selection required at least four clusters in the Central Calorimeter declared on-time or undecided. The two pairs with invariant mass closest to the $\pi^{\circ}$ mass were then fitted to $p \bar{p} \rightarrow \pi^{\circ} \pi^{\circ}$, with 4 energymomentum constraints plus two $\pi^{\circ}$ mass constraints, which give a $6 \mathrm{C}$-fit.

Events with fit probability bigger than $10^{-3}$ constitute the sample of $\pi^{\circ} \pi^{\circ}$ exclusive final state.

Fig. $6 a, b, c, d$ and e show the plots of $d \sigma^{M} / d\left|\cos \theta^{*}\right|$ (circles) for $E_{C M}=2.975,3097,3.526,3.556$ and $3.686 \mathrm{GeV}$ respectively. To compute 
do/d $\left|\cos \theta^{*}\right|$ we applied the correction $P_{\pi^{0} \pi^{0}}^{\pi^{0}}=\epsilon_{t} \times \epsilon_{f p} \times \epsilon_{a n} \times \epsilon_{\text {ace }}$, where $\epsilon_{\text {an }} \times \epsilon_{\text {ace }}=0.88$ at $E_{C M} \sim 2.985 \mathrm{GeV}$ and 0.82 at $\chi_{2}$ energy, and it is rather constant in $\cos \theta^{*}$. Recalling that $\epsilon_{i}=0.87$ and $\epsilon_{p}=0.87$, we obtained $P_{\pi^{0} \pi^{0}}^{\pi^{0}}=0.67$ at $E_{C M} \sim 2.985 \mathrm{GeV}$ and 0.62 at the $\chi_{2}$ energy. Finally, we subtracted the background mainly due to $3 \pi^{\circ}$ events with a $\pi^{\circ}$ escaping the detection that we estimated being $15 \%$ at $E_{C M} \sim 2.985 \mathrm{GeV}$ and $8 \%$ at the $x_{2}$ energy. (This estimation needs further investigation).

$d \sigma / d\left|\cos \theta^{*}\right|$ plots are reported (boxes) in Fig. 6a,b,c,d and e for $\left|\cos \theta^{m}\right|<0.5$. As stated before the range of $\cos \theta^{m}>0.5$ has to be treated with special care to define the fiducial volume for $\gamma$ detection.

The statistical errors are completely negligible and the quoted errors are dominated by the systematics. For all the cross sections values we estimated a $7 \%$ error, which comes from uncertainties on the efficiency determination and background estimation.

In Fig. 6a and d crosses represent $d \sigma / d \mid \cos \theta^{*} /$ from $R 704$ [7]. We notice that R704 results are systematically lower than E760 cross sections. Here we mention several causes of this systematic disagreement: overall luminosity scale, R704 fiducial volume correction (they had a 1/4 of $2 \pi$ azimuthal coverage), R704 overestimation (or E760 underestimation) of the efficiency due to trigger and analysis, and ET60 background, which has not correctly evaluated.

b) $\pi^{\circ} \gamma$ selection

As input we used the MLU4 DST's produced during the data taking.

The analysis was done in two steps:

step 1) entirely based on the ACP bits; a trigger had to satisfy the following requirements to pass the selection: a) $\leq 4$ clusters as reconstructed by the $A C P$; b) $E^{A C P}>0.9 E_{\text {tot }}$; ) $E_{\perp}^{A C P}>0.8 E_{\perp}$; d) highest invariant mass of a cluster pair $>2.0 \mathrm{GeV} / \mathrm{c}^{2}$.

This selection reduced the sample size of a factor $\sim 200$ at the $\eta_{c}$ with an efficiency $\epsilon_{f p}=0.93$.

step 2) We considered only triggers with $\leq 3$ clusters declared on time and at least 3 clusters when the clusters on time were summed to the undecided ones.

The cluster pairs giving an invariant mass $m_{\gamma \gamma}$ closest to the $\pi^{\circ}$ mass is assigned to the $\pi^{\circ}$. Triggers with $\left|m_{\gamma \gamma}-m_{\pi^{\circ}}\right|>100 \mathrm{MeV} / \mathrm{c}^{2}$ were rejected.

Finally, we fitted the events to $p \bar{p} \rightarrow \pi^{\circ} \gamma$ and cut to a fit probability of $10^{-3}$. Nominally this is a $5 C$ fit but in practice it is a $2 C$ fit being the error on the energy measurements much less constraining than the angu- 
lar ones. This observation suggests that a fraction of the $\pi^{0} \gamma$ sample could be due to $\pi^{\circ} \pi^{\circ}$ background. To evaluate the consistency of this fraction we considered the events with more than 3 clusters (the exceeding ones declared undecided) that were submitted to the $\pi^{\circ} \pi^{\circ}$ selection. Those fitting to the $\pi^{\circ} \pi^{\circ}$ hypothesis were removed from the $\pi^{\circ} \gamma$ sample. The fraction of events ambiguous to $\pi^{\circ} \pi^{\circ}$ and $\pi^{\circ} \gamma$ was $\sim 0.23$ indipendent on the center of mass energy. We add that requiring strictly 3 clusters the sample could have been reduced of $8 \%$ at the $\eta_{c}$ and $13 \%$ at $\chi_{2} .{ }^{1}$

In Fig. $7 \mathrm{a}$ and $\mathrm{b}$, we show the plot of $d \sigma_{\pi^{\circ} \gamma}^{M} / d \cos \theta_{\gamma}^{*}$ as a function $\cos \theta_{\gamma}^{*}$ for triggers satisfying the above conditions at $E_{C M}=2.985 \mathrm{GeV}$ and $3.556 \mathrm{GeV}$ respectively.

In eq. (2), the ferm $P_{\pi^{0} \pi^{0}}^{\pi^{0}} d \sigma_{\pi^{0} \pi^{0}} / d \cos \theta^{*}$ represents the fraction of events fitting to $\pi^{\circ} \gamma$ but coming from $\pi^{\circ} \pi^{\circ}$ leakage. Again, this is due to: a) one $\gamma$ was not detected (outside the acceptance or below detection threshold); b) two coalescent $\gamma$ 's were not separated by the cluster algorithm. The consistency of this contribution is plotted in Fig. $7 \mathrm{a}$ and $\mathrm{b}$ as shaded areas. We notice that the behavior of the measured cross section is different from the expected from $\pi^{\circ} \pi^{\circ}$, and this suggests a relevant contribution due to direct production of $\pi^{\circ} \gamma$.

Finally, to compute $d \sigma_{\pi^{0} \gamma} / d \cos \theta^{*}$ we evaluated $P_{\pi_{\alpha} \pi_{\gamma}}^{\pi_{\gamma}}=\epsilon_{t} \times \epsilon_{f p} \times$ $\epsilon_{a n} \times \epsilon_{a c c}$, where $\epsilon_{t}=0.89, \epsilon_{p p}=0.93$ and $\epsilon_{a n} \times \epsilon_{a c c}=0.93$ at $E_{C M}=2.985$ $\mathrm{GeV}$ and 0.89 at $\chi_{2}$ energy. $d \sigma_{\pi^{0} \gamma} / d \cos \theta^{*}$ from eq. (2) is plotted in Fig. 8a and $b$ for $E_{C M}=2.985 \mathrm{GeV}$ and $3.556 \mathrm{GeV}$ respectively.

In Fig. $9 \mathrm{a}$ and $\mathrm{b}$ we compare the $\pi^{\circ} \pi^{\circ}$ and $\pi^{\circ} \gamma$ cross section for $\left|\cos \theta^{*}\right|<0.5$ as function of $E_{C M}$. Parametrizing the cross section dependence on $E_{C M}$ as $\sigma=A \times E_{C M}^{B}$, we obtained $B=-18.2 \pm 5.2$ for $\pi^{\circ} \pi^{\circ}$ and $-15.2 \pm 2.4$ for $\pi^{\circ} \gamma$.

\section{c) $\gamma \gamma$ background estimation}

We refer to Eq. (3) to estimate $d \sigma_{\gamma \gamma}^{M} / d \cos \theta^{*}$. We make the obvious assumption that the contribution from continuum $\left(\sigma_{\gamma \gamma}\right)$ is negligible, and the only contributions are the terms representing the leakage from $\pi^{\circ} \pi^{\circ}$ and $\pi^{\circ} \gamma$. Before quoting the results we factorized the efficiencies due to the first pass selection and to the final analysis selection and assumed $\epsilon_{f p} \times \epsilon_{a n}=$ 1. Effectively, these contributions depend strongly on the choices made to extract the $\gamma \gamma$ sample. Nevertheless, we notice that $\epsilon_{f p} \times \epsilon_{a n}$ are typically between 0.8 and 0.9 . The distributions of $d \sigma_{\gamma \gamma}^{M} / d \mid \cos \theta^{*}$ as a function of $\cos \theta^{*}$ are reported in Fig. $10 \mathrm{a}$ and $b$, respectively at the $\eta_{c}$ and $\chi_{2}$ energies.

\footnotetext{
${ }^{1}$ Even though the leakage from other channels (as for instance $\pi^{0} \eta$ ) is expected to be negligible, we are now investigating those contributions.
} 
Summing the differential cross section up to $\left|\cos \theta^{*}\right|=0.2(0.4)$ at the $\eta_{c}\left(\chi_{2}\right)$, we estimated the expected $\sigma_{\gamma \gamma}$ background, as expected from $\pi^{\circ} \pi^{\circ}$ and $\pi^{\circ} \gamma$ leakage. We obtained $\sigma_{\gamma \gamma}=39.1 \mathrm{pb}$ at the $\eta_{c}$ energy and $24.1 \mathrm{pb}$ at $E_{C M}=$ $3.556 \mathrm{GeV}$.

\section{Conclusions}

We compared the measured $\pi^{\circ} \gamma$ cross section to the expected from the $\pi^{\circ} \pi^{\circ}$ leakage due to: a) one $\gamma$ escaped detection because out of acceptance; b) two coalescemt $\gamma^{\prime}$ 's from a symmetric $\pi^{\circ}$ decay where reconstructed as a single $\gamma$. We observed a large discrepancy between the measured and the expected cross section (see Fig. 7.a-b). The obvious explanation invokes that a consistent contribution comes from a continuum production of $\pi^{\circ} \gamma$. We obtained $\sigma_{\pi^{\circ} \sim}$ ranging from $\sim 26$. nb at $E_{C M}=2.985 \mathrm{GeV}$ to $1 . n b$ at $E_{C M}=3.556 \mathrm{GeV}$ for an angular acceptance limited to $\left|\cos \theta^{*}\right|<0.5$.

Summing the contributions of $\pi^{0} \pi^{\circ}$ and $\pi^{\circ} \gamma$, we estimated a $\gamma \gamma$ background of $\sim 39.1 \mathrm{pb}$ at at $E_{C M}=2.985 \mathrm{GeV}$ for $\left|\cos \theta^{*}\right|<0.20$ and $\sim 24.1 \mathrm{pb}$ at at $E_{C M}=3.556 \mathrm{GeV}$ for $\left|\cos \theta^{*}\right|<0.4$.

\section{References}

[1] G. Wolf, Proceedings of the 1971 International Symposium on Electron and Photon Interaction at High Energies pg. 189; and references therein.

[2] M. A. Ming et al., Nucl. Phys. B 51, (1977) 77.

[3] Katz et al., Phys. Rev. Lett. 19, (1967) 265.

[4] E760 Collaboration, Measurement of the $\gamma \gamma$ Partial Width of the $\chi_{2}$ Charmonium Resonance, to be published.

(5) Most of the contribution to this Monte Carlo comes from A. Ceccucci and M. Sozzi

16] The parametrization used is the same as the one implemented to compute the shower direction in the offline clustering algorithm (see Subroutine DOITALL).

[7] C. Baglin et al., Nucl. Phys. B 368 (1992) 175. 
Fig. 1 Total reconstructed energy distribution for $p \bar{p}-\pi^{0} \pi^{\circ}$ events at $E_{C M}=2.985 \mathrm{GeV}:$ a) Monte Carlo; b) data.

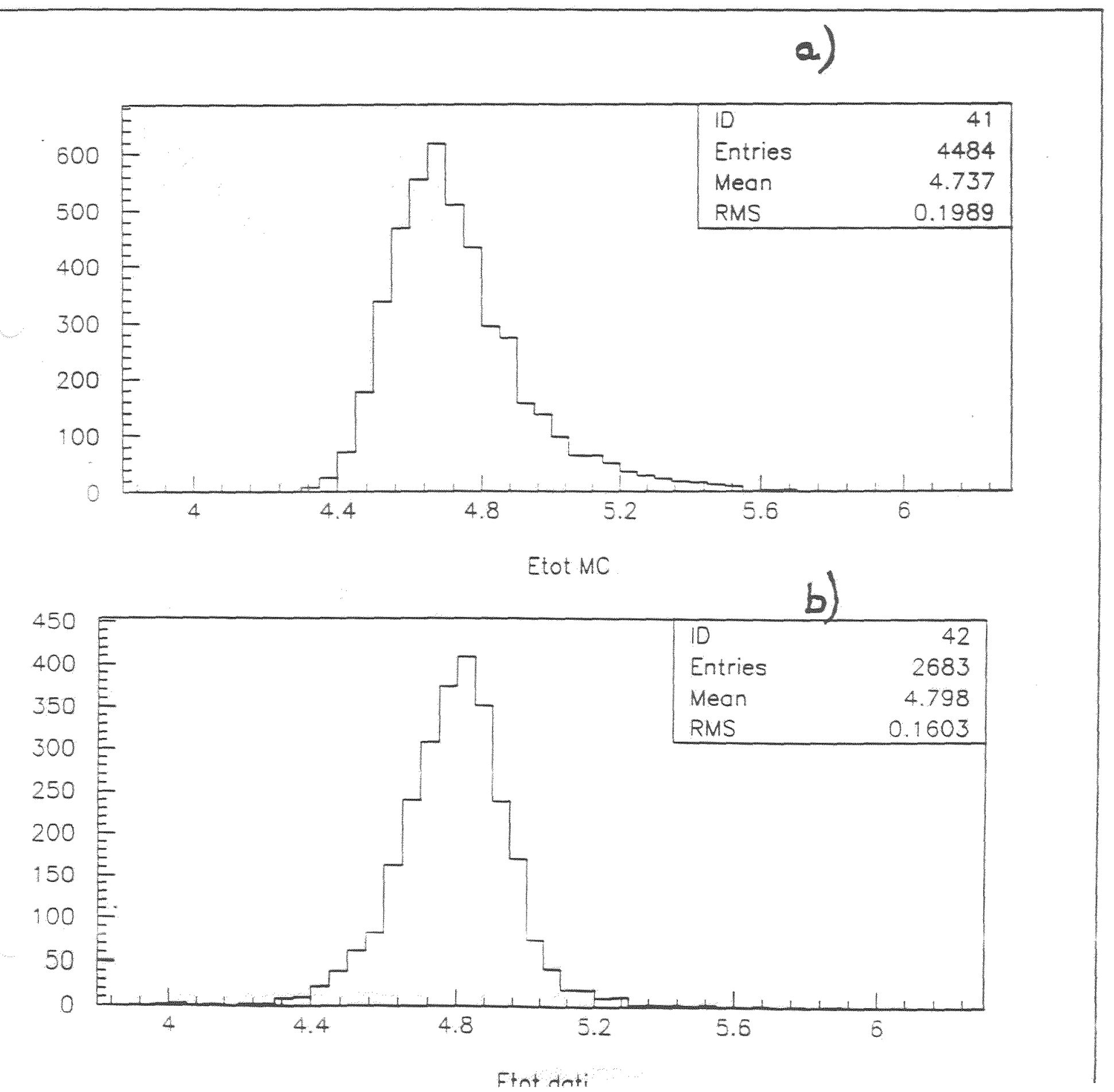


Fig. 2 Distribution of the difference between the reconstructed and fitted $\gamma$ polar angle : a) Monte Carlo; b) data.

a)
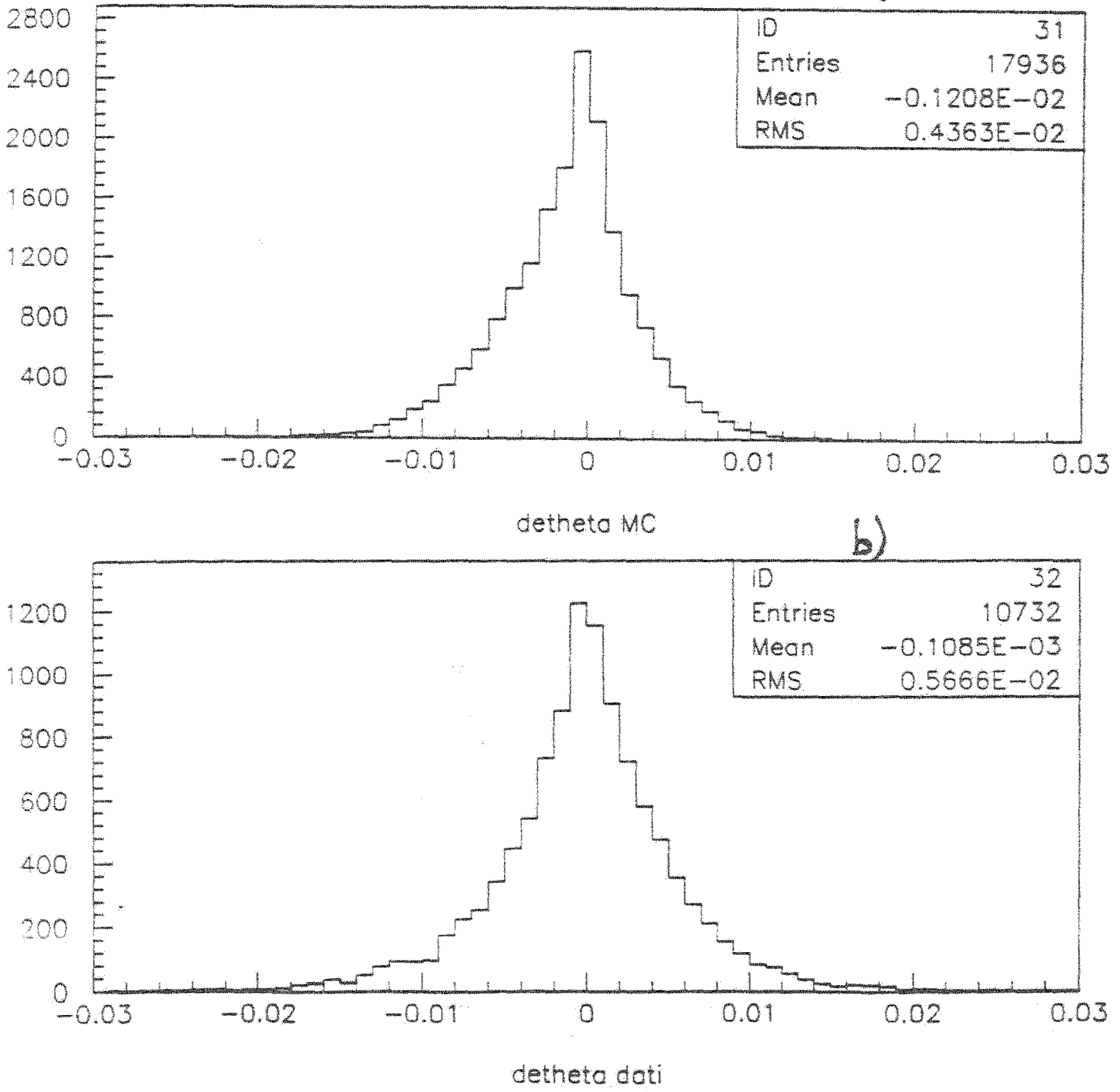
Fig. 3 Distribution of the difference between the reconstructed and fitted $\gamma$ azimuthal angle : a) Monte Carlo; b) data.

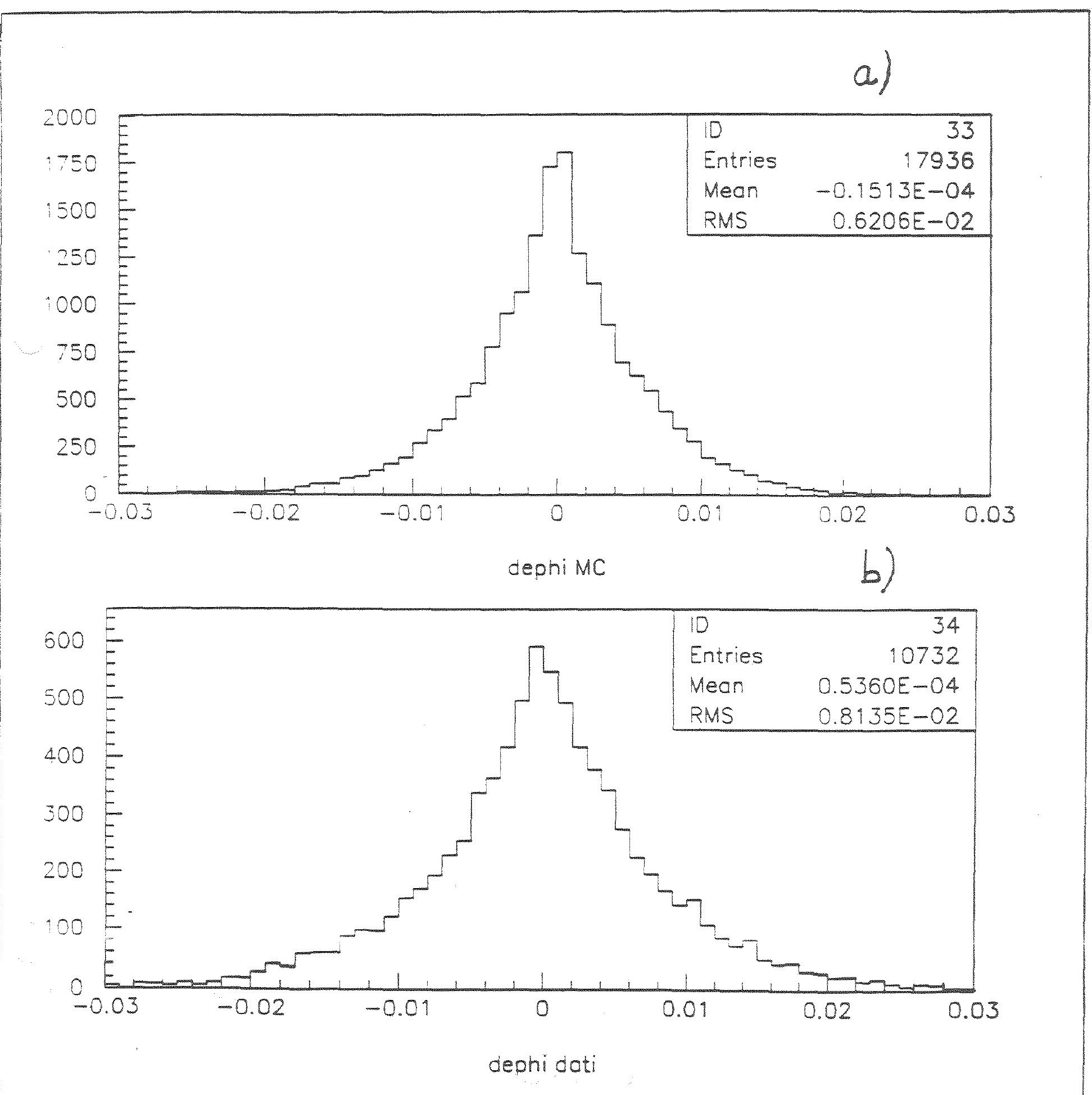


Fig. 4 Cluster mass distribution in $p \bar{p}-\pi^{\circ} \pi^{\circ}$ events at $E_{C M}=2.985 \mathrm{GeV}$ a) Monte Carlo; b) data.

The promizent peak is from $\pi^{\circ}$ symmetric decay, while the shoulder on the left is due to isolated $\gamma$ clusters.

\section{a)}

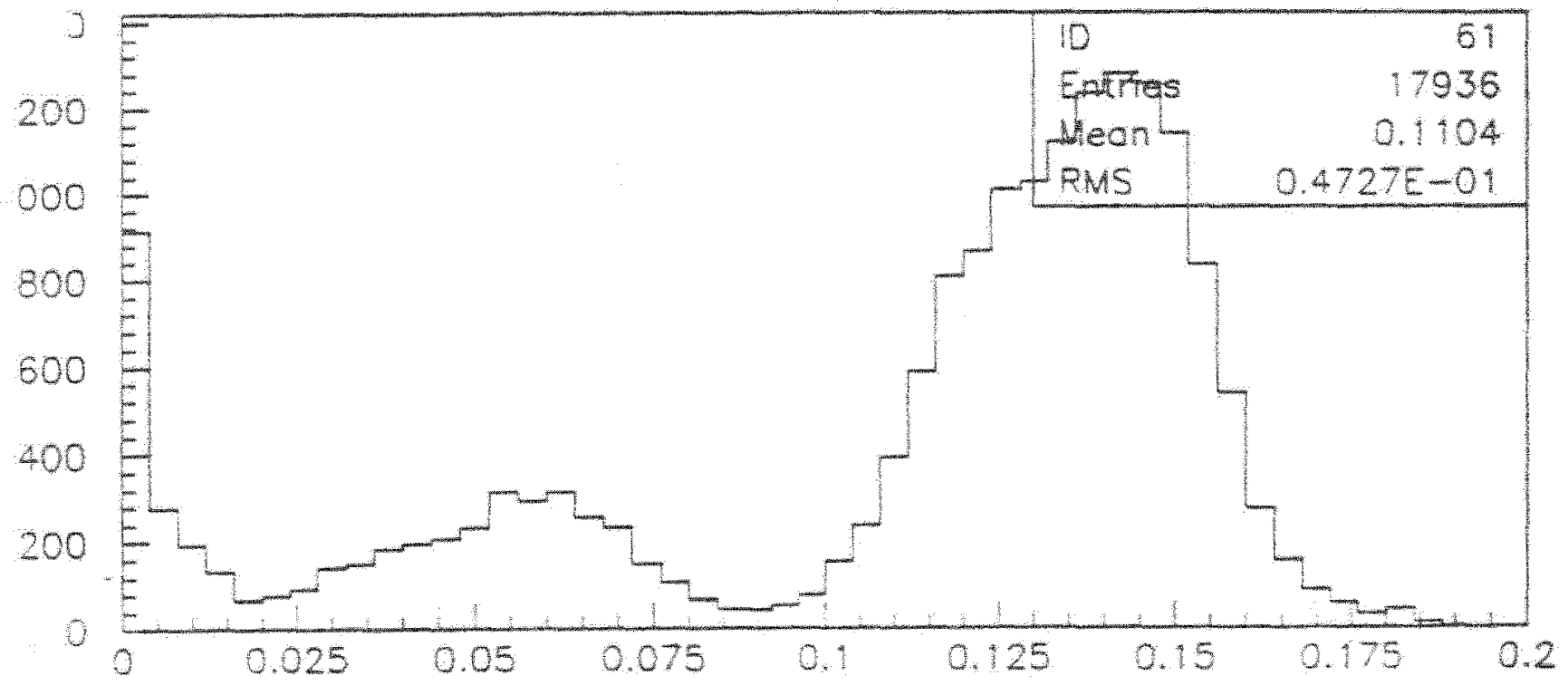

cluster moss MC

b)

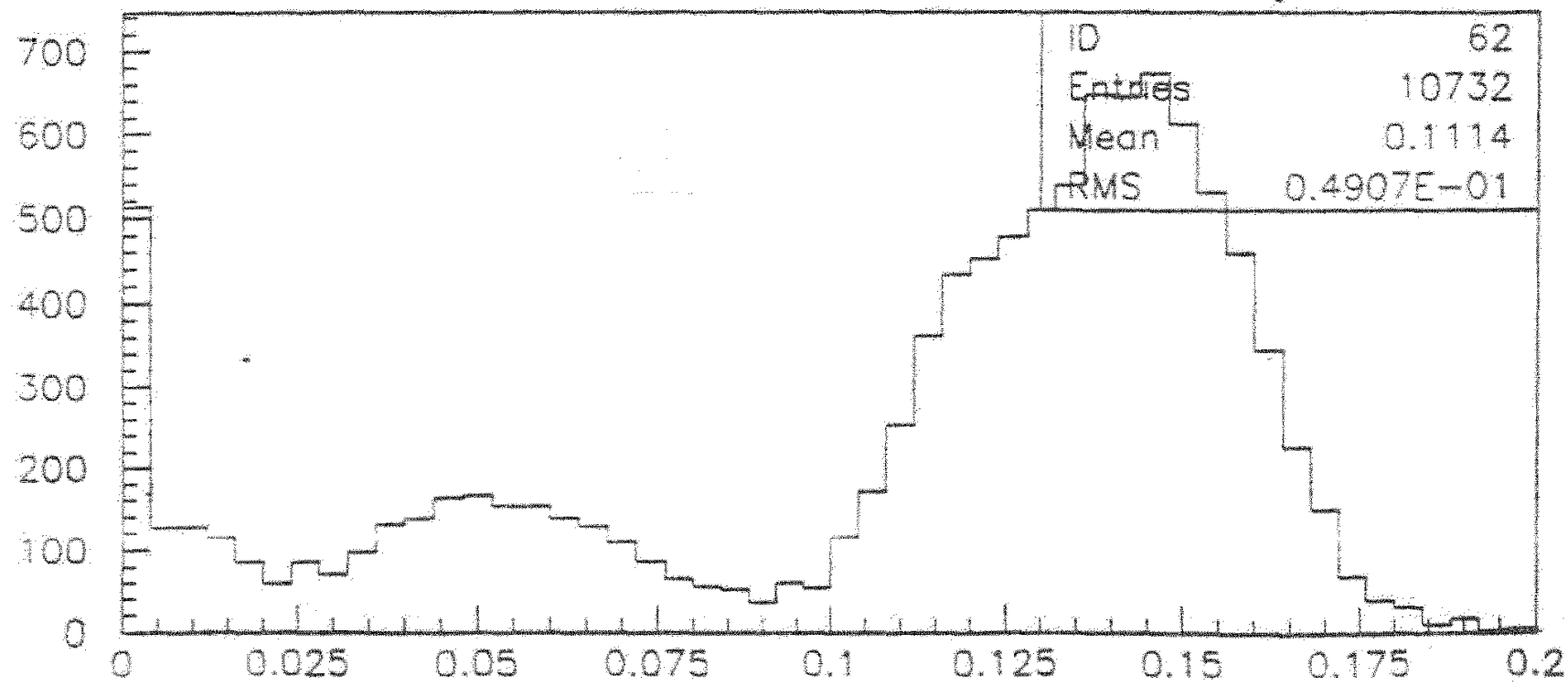


Fig. $5 \pi^{0} \rightarrow \gamma \gamma$ invariant mass distribution : a) Monte Cario; b) data.

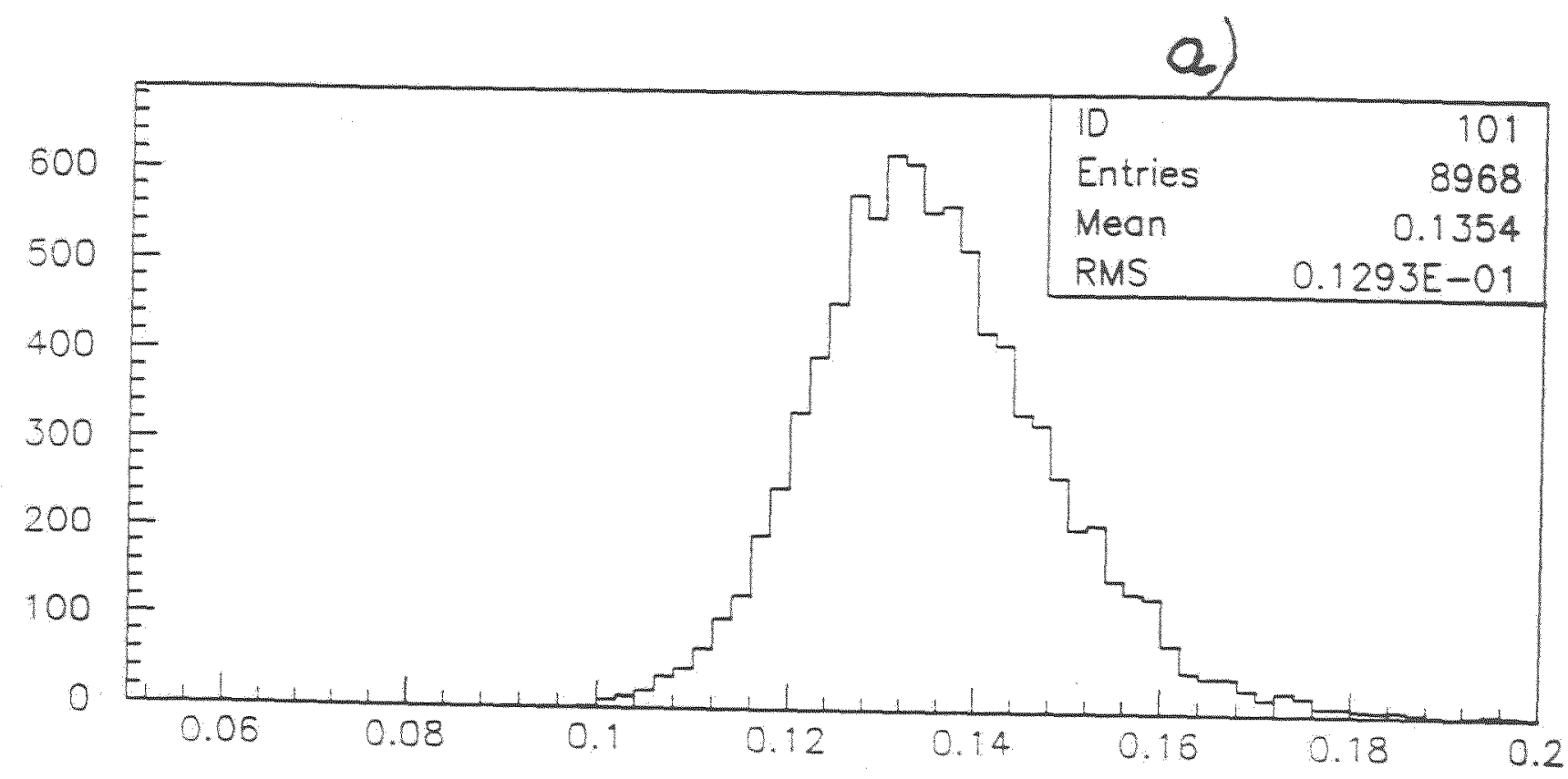

Mgg MC

b)

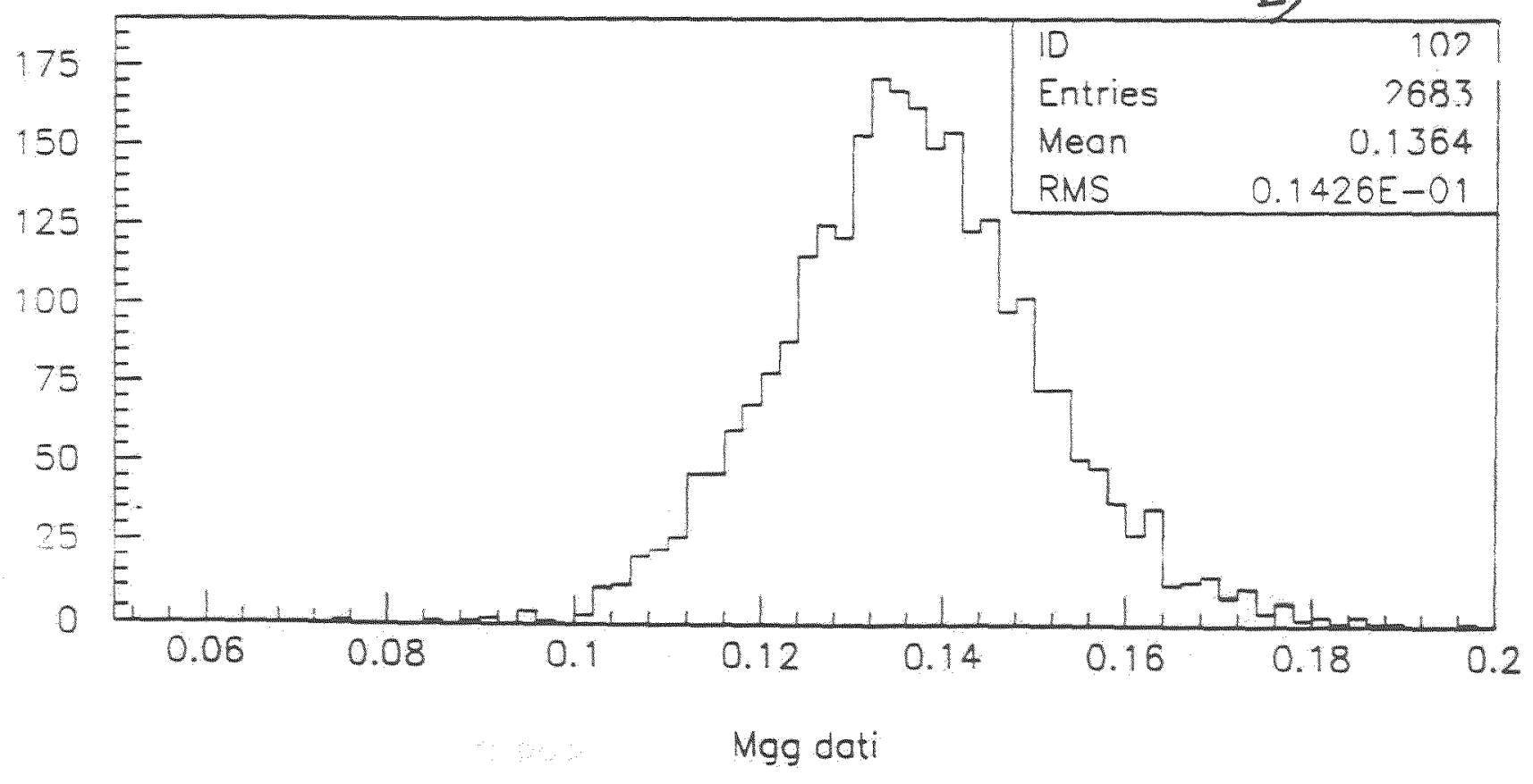


Fig. 6.a $p \bar{p}-\pi^{\circ} \pi^{\circ}$ cross section vs. $\left|\cos \theta^{*}\right|$ at $E_{C M}=2.975 \mathrm{GeV}$ :。 correspond to the measured cross sections and $\square$ to the corrected ones (see text); crosses correspond to R704 results.

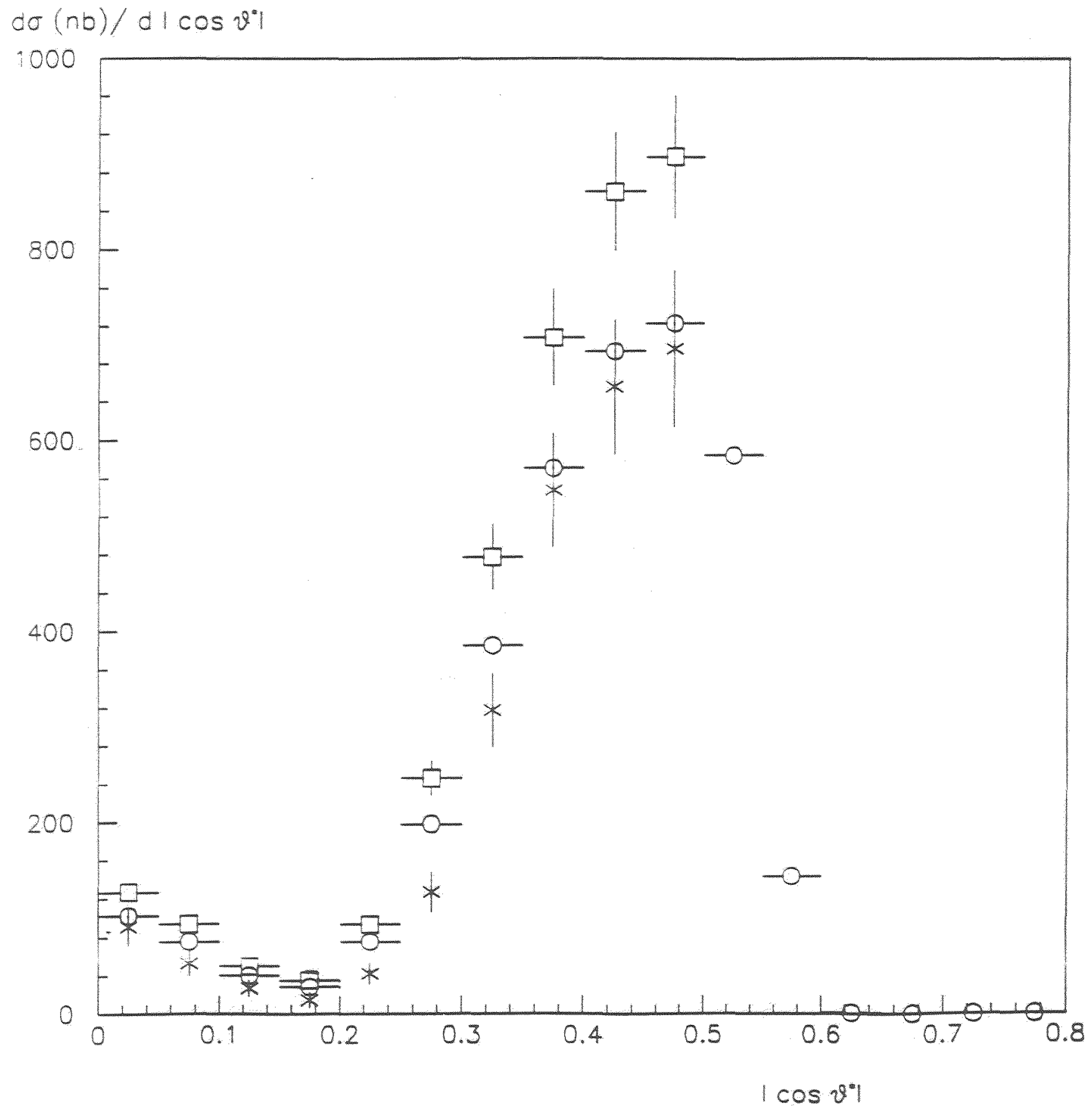


Fig. G.b $p \ddot{p}-\pi^{\circ} \pi^{\circ}$ cross section vs. $\left|\cos \theta^{\prime \prime}\right|$ at $E_{C M}=3.097 \mathrm{GeV}: 0$ correspond to the measured cross sections and $\square$ to the corrected ones (see text).

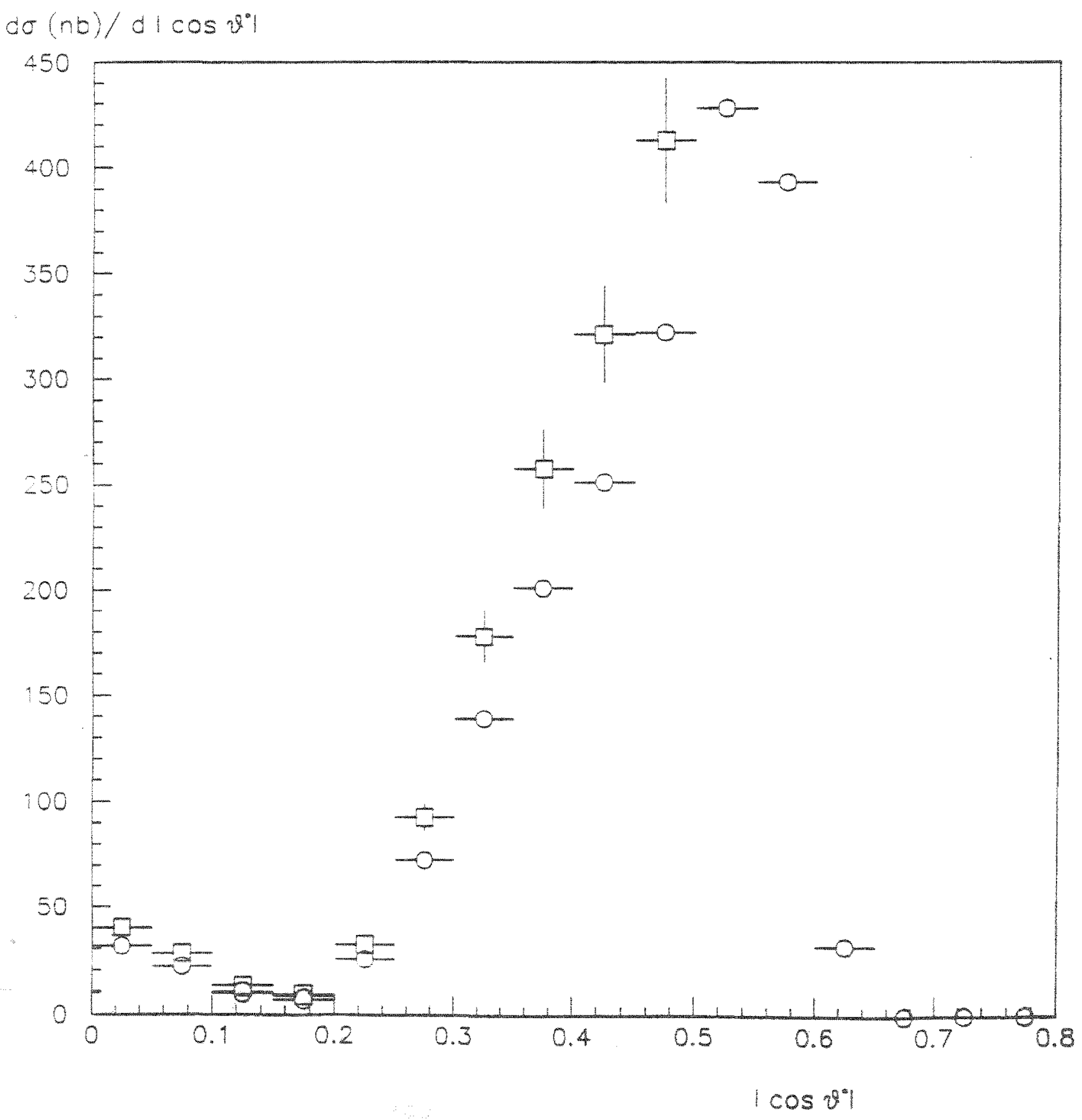


Fig. 6.c $p \bar{p}-\pi^{\circ} \pi^{\circ}$ cross section vs. $\left|\cos \theta^{*}\right|$ at $E_{C M}=3.525 \mathrm{GeV}: 0$ correspond to the measured cross sections and $\square$ to the corrected ones (see text).

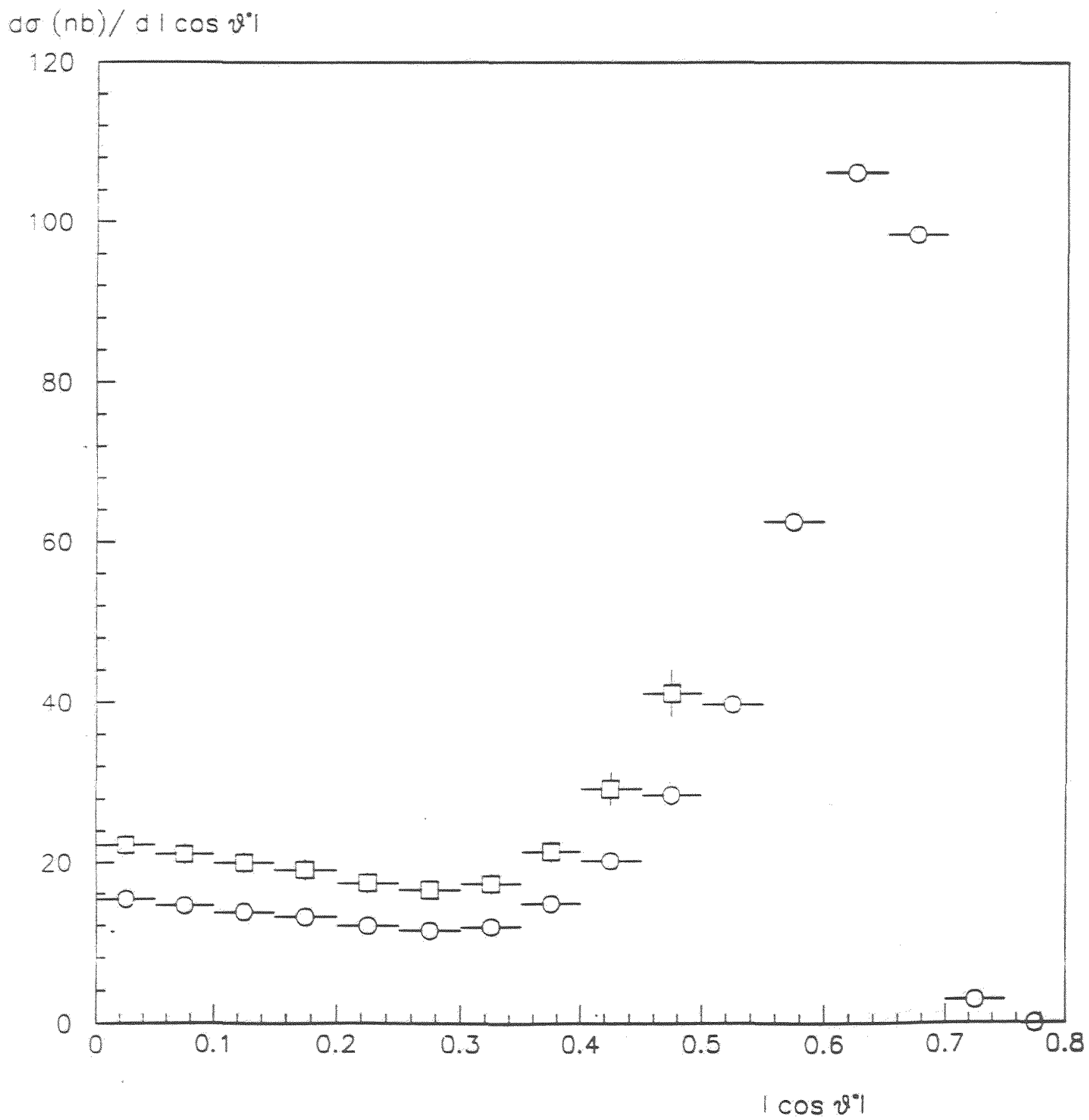


Fig. 6.d $p \bar{p} \rightarrow \pi^{\circ} \pi^{\circ}$ cross section vs. $\left(\cos \theta^{*} \mid\right.$ at $E_{C M}=3.556 \mathrm{GeV}: 0$ correspond to the measured cross section and $\square$ to the corrected ones (see text).

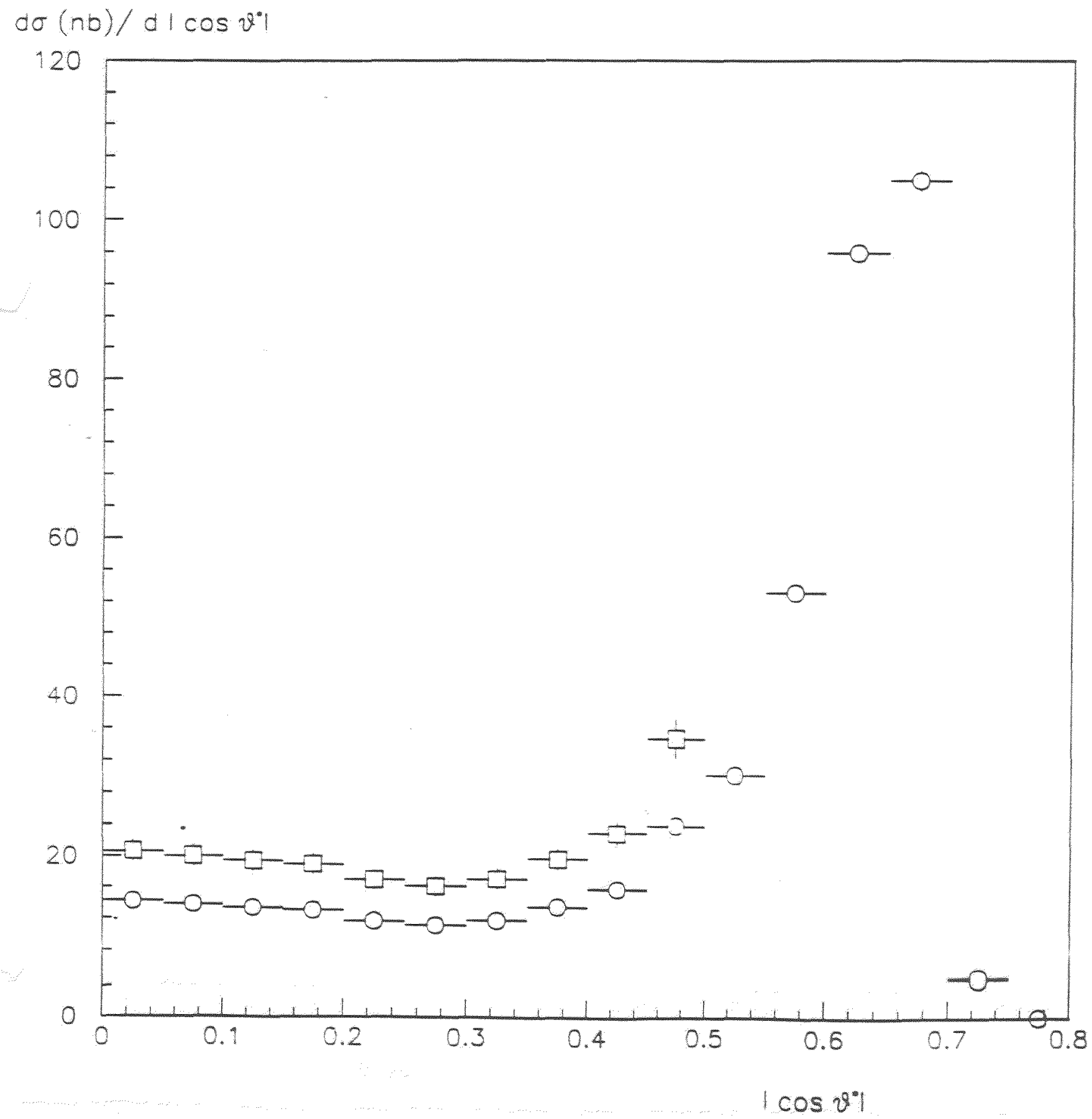


Fig. 6.e $p \bar{p}-\pi^{\circ} \pi^{\circ}$ cross section vs. $\left|\cos \theta^{*}\right|$ at $E_{C M}=3.686 \mathrm{GeV}: 0$ correspond to the measured cross section and $\square$ to the corrected ones (see text); crosses correspond to R704 results.

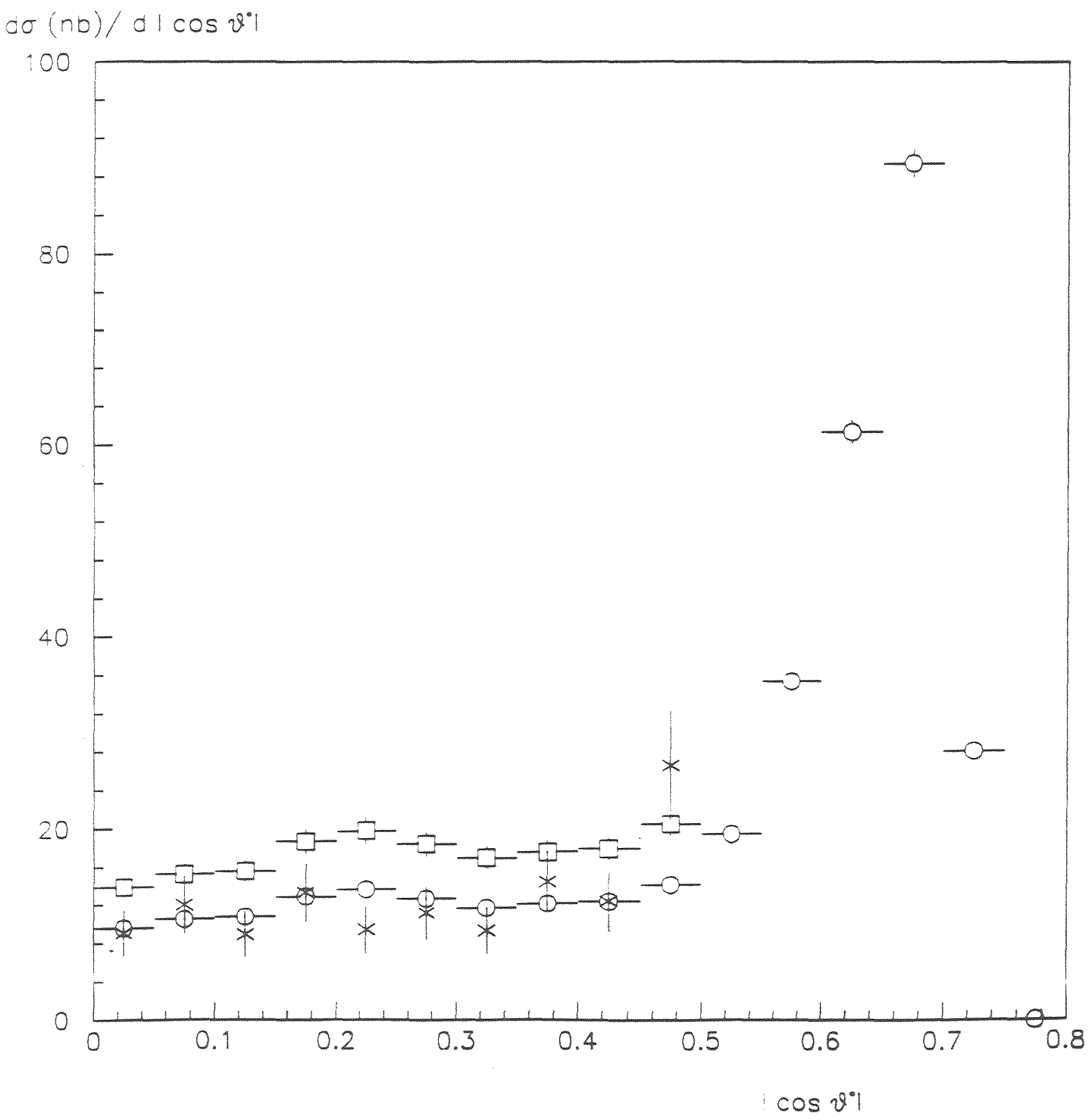


Fig. 7.a $p \bar{p}-\pi^{\circ} \gamma$ measured cross section ys. $\cos \theta_{\gamma}^{*}$ at $E_{C M}=2.985$ $\mathrm{GeV}$. Insert shows the central region of the distribution $\left(\left|\cos \theta_{\%}^{*}\right|<0.3\right)$. The shaded aress represent the expected contribution from $\pi^{0} \pi^{0}$ leairge.

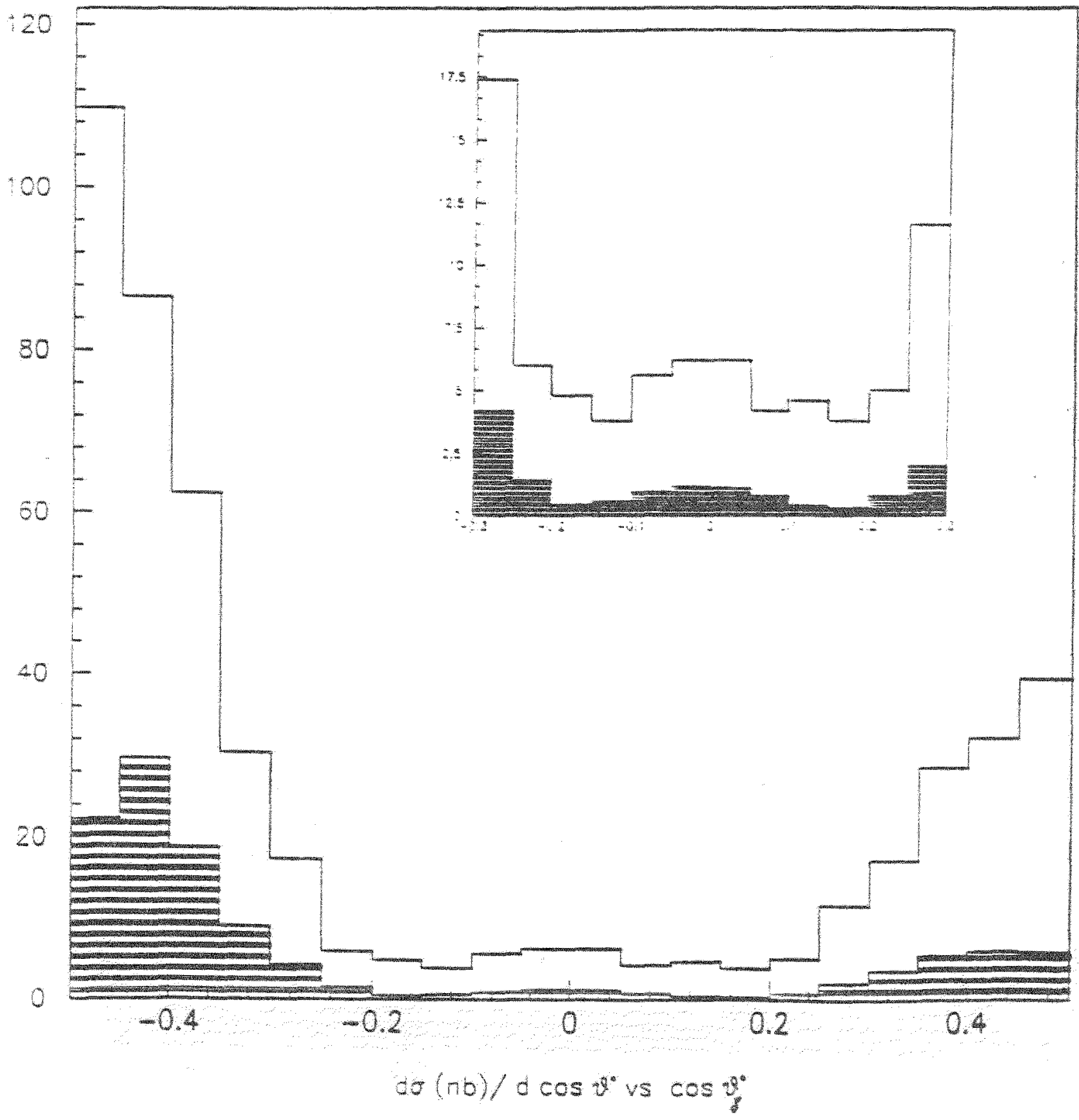


Fig. 7.b $p \bar{p} \rightarrow \pi^{\circ} \gamma$ measured cross section vs. $\cos \theta_{\gamma}^{m}$ at $E_{C M}=3.556 \mathrm{GeV}$. The shaded area represents the expected contribution from $\pi^{\circ} \pi^{\circ}$ leakage.

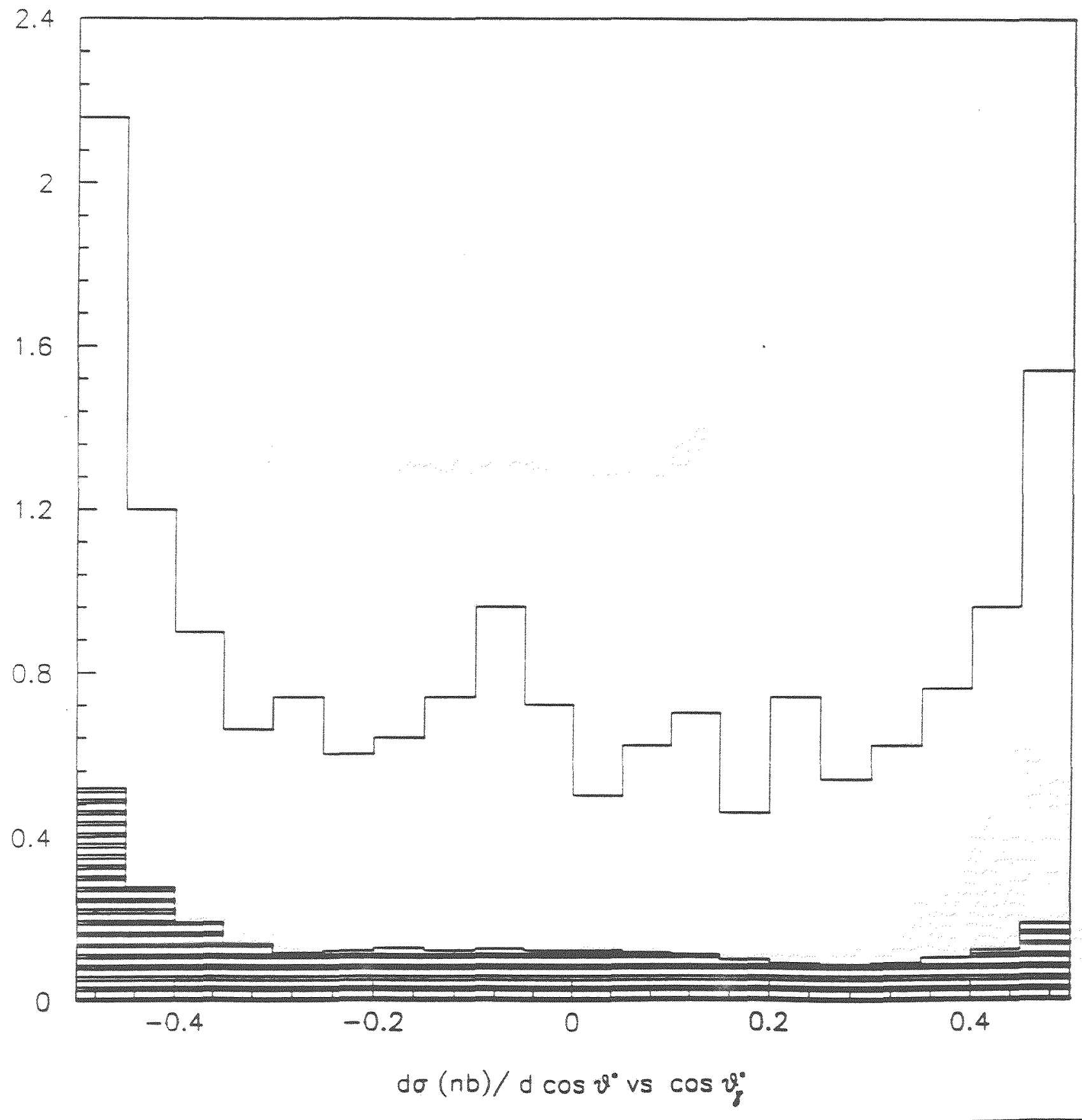


Fig. 8.a-b $p \bar{p} \rightarrow \pi^{\circ} \gamma$ cross section vs. $\cos \theta_{\gamma}^{*}$ at a) $E_{C M}=2.985 \mathrm{GeV}$, b) $E_{C M}=3.556 \mathrm{GeV}$. The cross section is corrected for efficiencies after the subtraction of the expected leakage from $\pi^{\circ} \pi^{\circ}$
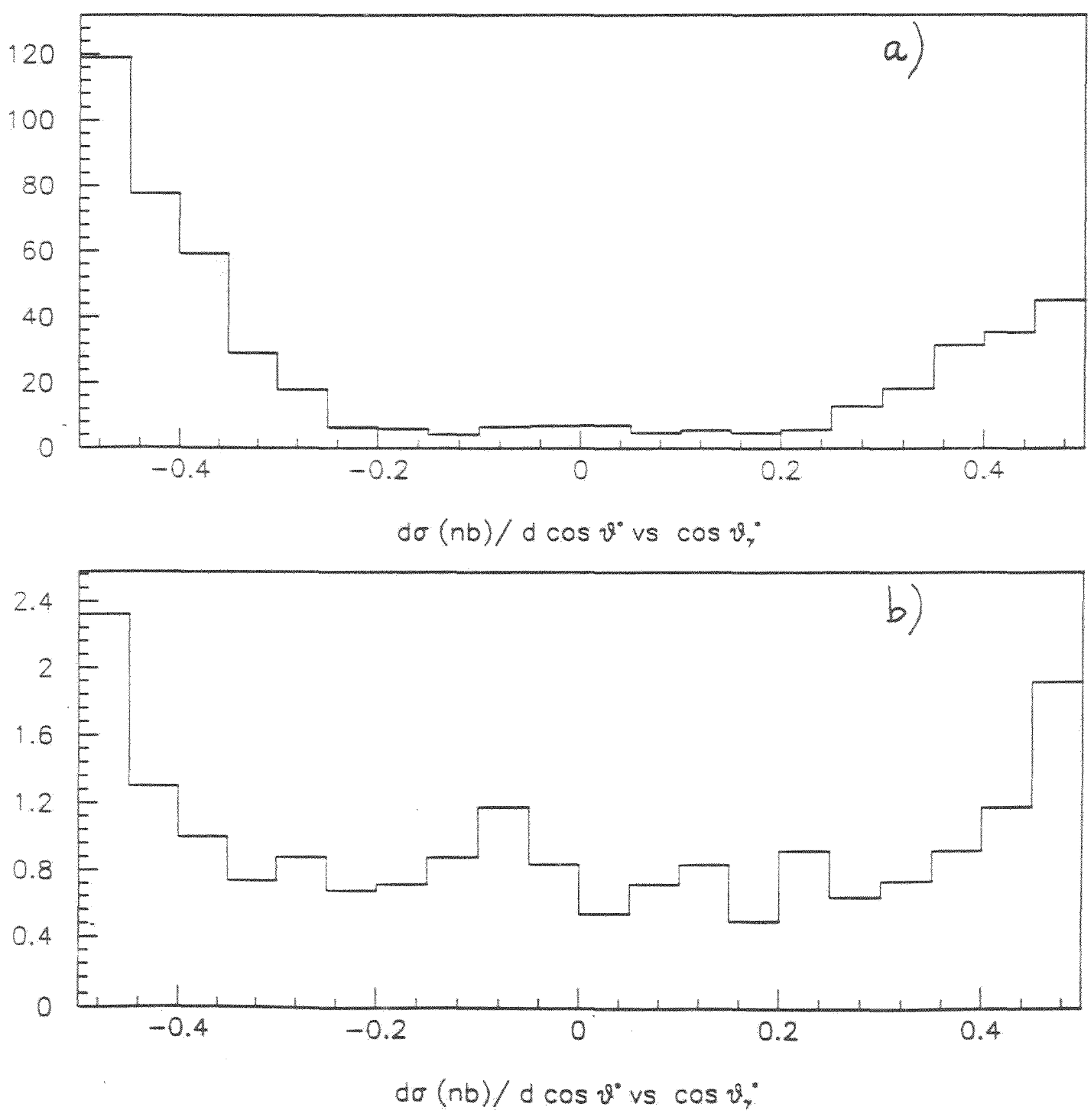
Fig. 9.aub Corrected cross section vs. $E_{C M}$ for $\left.\left.a\right): p \vec{p} \rightarrow \pi^{a} \pi^{\circ} ; b\right): p \bar{p} \rightarrow \pi^{0} \gamma$ in the $\left|\cos \theta_{\gamma}^{*}\right|<0.5$ range,
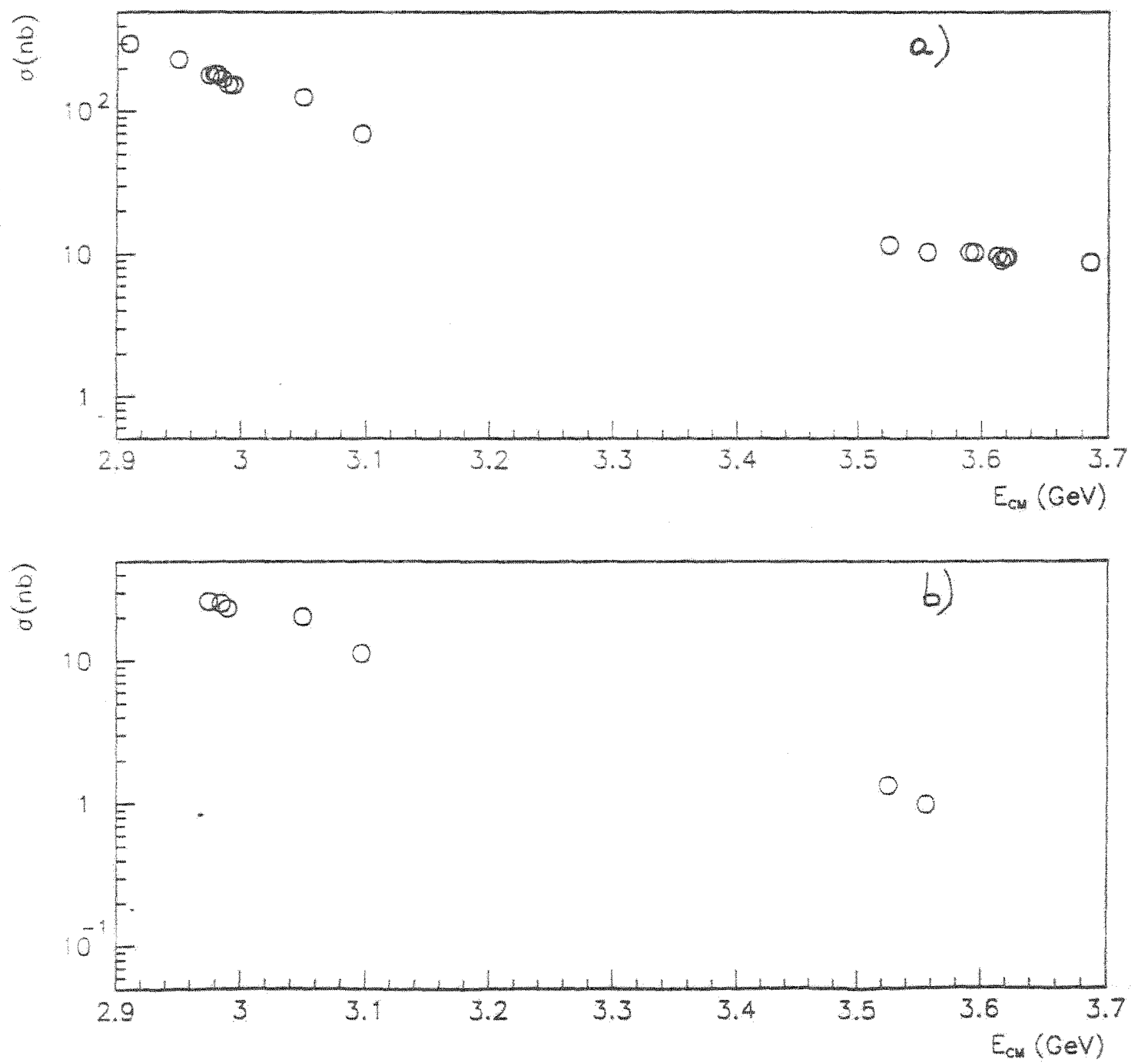
Fig. 10.a-b $\gamma \gamma$ background cross section vs. $\cos \theta^{*}$ for $\cos \theta^{*}$ bins of 0.05 a) refers to $E_{C M}=2.985 \mathrm{GeV}$, and b) to $E_{C M}=3.556 \mathrm{GeV}$.
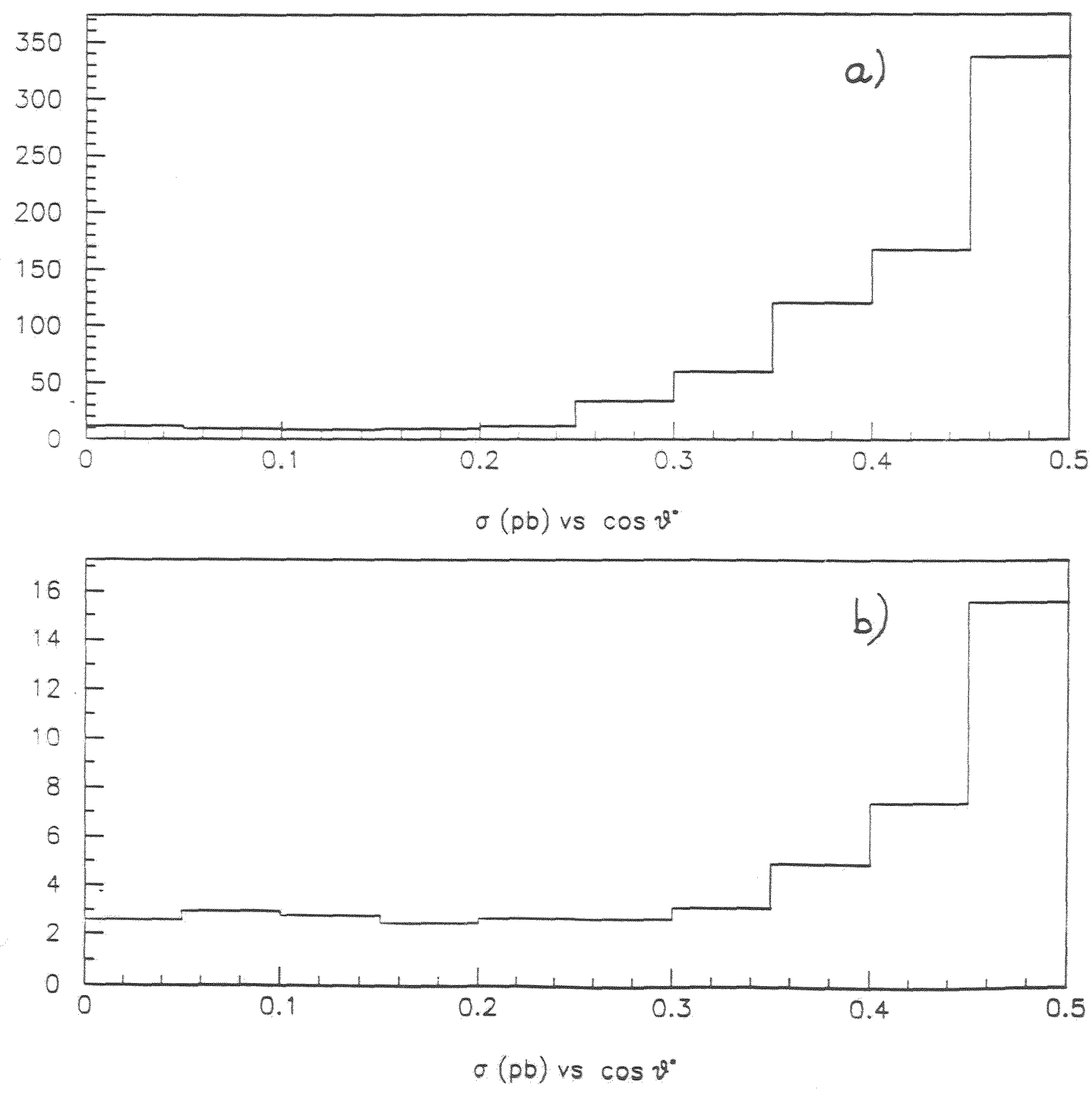
